\title{
Biometric Method Based On The Matching Of Dilated And Skeletonized Ir Images Of The Veins Map Of The Dorsum Of The Hand
}

\author{
W. Alejo, D. Rodríguez and G. Kemper
}

\begin{abstract}
This work proposes a biometric identification system that works together with a palm vein reader sensor and a hand-clenching support, designed to perform the capture the back of the hand. Several processing steps were performed: extraction of the region of interest, binarization, dilation, noise filtering, skeletonization, as well as extraction and verification of patterns based on the measurment of coincidence of vertical and horizontal displacements of skeletonized and dilated images. The proposed method achieved the following results: processing time post capture of 1.8 seconds, FRR of $0.47 \%$ and FAR of $0,00 \%$, with a referential database of 50 people from a total of 1500 random captures.
\end{abstract}

Keywords - biometrics, FAR, FRR, ROI, identification, pattern recognition, veins patterns, dorsum of the hand.

\section{INTRODUCCIÓN}

$\mathrm{E}_{\mathrm{d}}^{\mathrm{N}}$ $\mathrm{N}$ los últimos años ha existido un gran interés por descubrir y mejorar los sistemas electrónicos biométricos, con énfasis en aquellos considerados no invasivos.

Esto también ha sido motivado por el creciente interés de encontrar nuevas formas de protección para el acceso a la información clasificada, los espacios y las zonas restringidas.

En la actualidad, los sistemas de seguridad de información y acceso como las claves electrónicas, las tarjetas magnéticas, el RFID (Radio Frequency IDentification), el "chip card" entre otros, permiten que el acceso sea restringido hasta un cierto nivel de protección, ya que estos sistemas no requieren de la presencia física de la persona autorizada (pues bastaría con poseer la clave o el medio físico para lograr el acceso).

Ante dicha problemática, la ingeniería biométrica ha desarrollado sistemas en los cuales es necesario contar con la presencia de la persona autorizada, a fin de validar el acceso correspondiente. Entre ellos se puede destacar a los sistemas de reconocimiento por huellas dactilares, los sistemas de reconocimiento por rostro, sistemas de voz, iris entre otros.

En todos los métodos se busca siempre robustez, confiabilidad, practicidad, comodidad (procurando ser lo menos invasivo posible) y rapidez en el tiempo de respuesta y validación. Ante ello, el método biométrico de reconocimiento por el mapa de venas del dorso de la mano, ofrece las ventajas de no ser invasivo a la persona y tener poca alteración en el tiempo (debido a que las venas no están externamente expuestas). Además presenta una robustez muy cercana a la de las huellas dactilares, tal como será demostrado en los

W. Alejo, Universidad Peruana de Ciencias Aplicadas, Lima, Perú, willy.alejo@gmail.com

D. Rodríguez, Universidad Peruana de Ciencias Aplicadas, Lima, Perú, danielr.carhz@gmail.com

G. Kemper, Universidad Peruana de Ciencias Aplicadas, Lima, Perú, guillermo.kemper@gmail.com resultados obtenidos en el presente trabajo.

La Tabla I [1] muestra un análisis comparativo general de las tecnologías biométricas más importantes.

TABLA I. ANÁLISIS COMPARATIVO GENERAL DE VARIAS TECNOLOGÍAS BIOMÉTRICAS [1].

\begin{tabular}{|c|c|c|c|c|c|}
\hline $\begin{array}{c}\text { Tipo de } \\
\text { Biometría }\end{array}$ & Precisión & Costo & $\begin{array}{c}\text { Tamaño } \\
\text { del Patrón }\end{array}$ & $\begin{array}{c}\text { Estabilidad } \\
\text { en el } \\
\text { Tiempo }\end{array}$ & $\begin{array}{c}\text { Nivel de } \\
\text { Segurid } \\
\text { ad }\end{array}$ \\
\hline Rostro & Bajo & Alto & Grande & Bajo & Bajo \\
\hline Iris & Alto & Alto & Pequeño & Medio & Medio \\
\hline $\begin{array}{c}\text { Huella } \\
\text { Dactilar }\end{array}$ & Medio & Bajo & Pequeño & Bajo & Bajo \\
\hline $\begin{array}{c}\text { Mapa de } \\
\text { Venas }\end{array}$ & Alto & Medio & Medio & Alto & Alto \\
\hline Voz & Bajo & Medio & Pequeño & Bajo & Bajo \\
\hline Labios & Medio & Medio & Pequeño & Medio & Alto \\
\hline
\end{tabular}

Para el caso del mapa de venas del dorso de la mano, algunos autores han realizado diferentes propuestas que involucran el proceso de adquisición de la imagen, algoritmos de pre-procesamiento, algoritmos de reconocimiento y métodos de validación de usuarios.

Motato y Loaiza en [2] proponen por ejemplo un método de extracción de venas basado en un algoritmo de umbralización adaptiva, en el que se asigna un valor de umbral para cada pixel en función de los niveles de gris de sus vecinos. Estos umbrales corresponden al valor promedio de los niveles de gris de una vecindad de "N x N" alrededor de cada pixel. El método presenta deficiencias cuando las sombras en la imagen adquirida eliminan algunas venas y afectan la conectividad entre ellas (generando regiones muy pequeñas con distorsión). También proponen un método de extracción de descriptores basado en la generación de subimágenes que contienen los cruces horizontales y verticales de la imagen binarizada esqueletizada. Sin embargo presenta algunas limitaciones en el tamaño de la grilla de submuestreo utilizada.

Chen et al. en [3] explican por otro lado que el método de Line-segment Hausdorff Distance (LHD) presenta buenos resultados en varias tecnologías biométricas, pero en lo referente a la información de la estructura de venas de la mano, no es tan claro y preciso (como el caso de huellas dactilares o palmares), de modo que este método no es una buena opción para el reconocimiento de venas. Además, indican que los métodos desarrollados para el reconocimiento de patrones vasculares o la ubicación de venas como Point-to-Point 


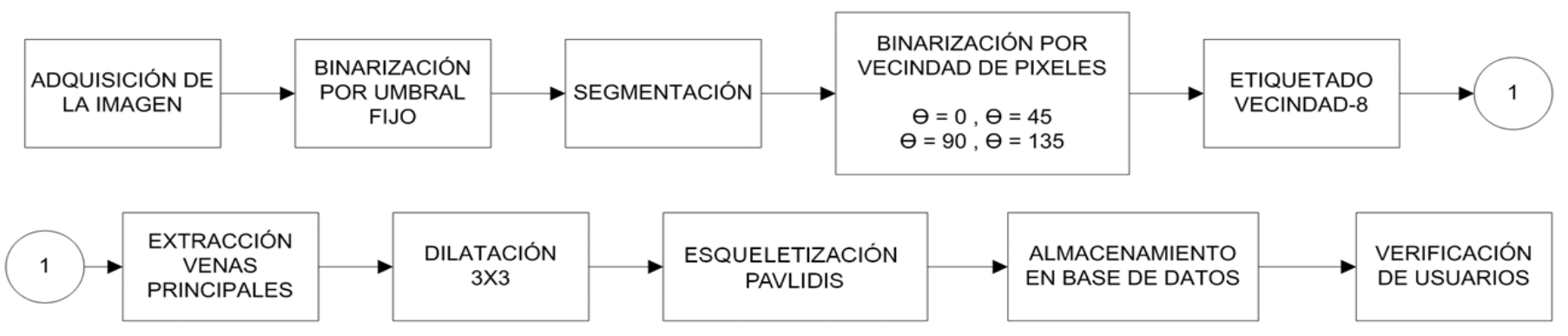

Figura 1. Diagrama de bloques del método propuesto.

Matching (P2PM), Similarity-based Mix Matching (SMM) e Iterative Closest Point Matching (ICPM), sufren problemas de rotación y desplazamiento de la imagen, así como en la extracción de patrones de verificación. Esto presenta mayor sensibilidad en la palma, en la parte inferior de la muñeca y en el dorso de la mano.

Zhang et al. proponen en [4] un método de extracción de venas mediante 4 filtros Gausianos que buscan extraer venas en distintas direcciones: vertical, horizontal, diagonal $45^{\circ}$ y diagonal $135^{\circ}$. En comparación a otros métodos, los filtros propuestos por Zhang et al. son menos vulnerables a la distorsión y ruido que presenta la imagen original. Ellos realizaron este análisis para imágenes de la palma de la mano, sin embargo existe casos donde las venas se pueden mezclar con los dermatoglifos.

En cuanto al método de comparación, Zhang et al. proponen la comparación punto a punto entre las imágenes binarizadas. La deficiencia de este método es que alcanza una tasa FAR (Tasa de Falsa Aceptación) de 5.5\% con tan solo 24 usuarios registrados.

En ese contexto, el método de segmento de distancia de línea de Hausdorff (LHD) aplicado al mapa de venas de la palma de la mano tiene mejores resultados experimentales. Sin embargo, la información de la estructura de venas de la palma no es tan clara como las venas del dorso.

Cabe resaltar que métodos como el P2PM, LHD y MHD (Modified Hausdorff Distance), son afectados por la rotación y el desplazamiento que puede sufrir la imagen adquirida.

Se hace necesario por tanto explotar y desarrollar nuevas alternativas de procesamiento y extracción de características, a fin de robustecer y mejorar el desempeño de los métodos y sistemas biométricos basados en el mapa de venas del dorso de la mano. En ese sentido, el presente trabajo propone un método alternativo basado en la medida de coincidencia de los desplazamientos verticales y horizontales de las imágenes esqueletizadas (obtenidas de la captura) y las imágenes dilatadas del mapa de venas de la región dorsal de la mano almacenadas previamente en una base de datos (por cada usuario registrado).

El método propuesto alcanzó una tasa de falso rechazo (FRR) de $0,47 \%$ y una tasa de falsa aceptación (FAR) de $0,00 \%$. Además de ello, el tiempo de procesamiento $\mathrm{y}$ validación de un usuario fue menor a 1.8 segundos.
Cabe resaltar que sistemas biométricos similares comercializados y patentados por Hitachi (analiza la parte posterior de la mano) y Fujitsu (analiza la palma de la mano) aseguran una tasa de FRR (Tasa de Falso Rechazo) y una tasa FAR de cerca del $0 \%$ en 140000 manos analizadas. Sin embargo no detallan ni la base de datos ni los métodos de validación utilizados en la obtención de estos resultados.

En las siguientes secciones del presente documento se detallarán el método propuesto, la validación y los resultados obtenidos comparados con otros métodos biométricos.

\section{Descripción Del Método Propuesto}

El diagrama de bloques del método propuesto es mostrado en la Figura 1. Las etapas de procesamiento desde la captura de la imagen hasta la identificación del usuario, serán descritas en las siguientes secciones.

\section{A. Adquisición de la imagen}

Para la adquisición de las imágenes fue utilizado un sensor PALMVEIN READER, el cual es ubicado en el recinto de adquisición de imágenes mostrado en la Fig. 2. El recinto ofrece una estructura interna para que el usuario ingrese la mano y luego la cierre apretando un eje con guías para los dedos. De esa manera, se forma un puño con la parte dorsal expuesta directamente hacia el sensor para la posterior adquisición de la imagen.

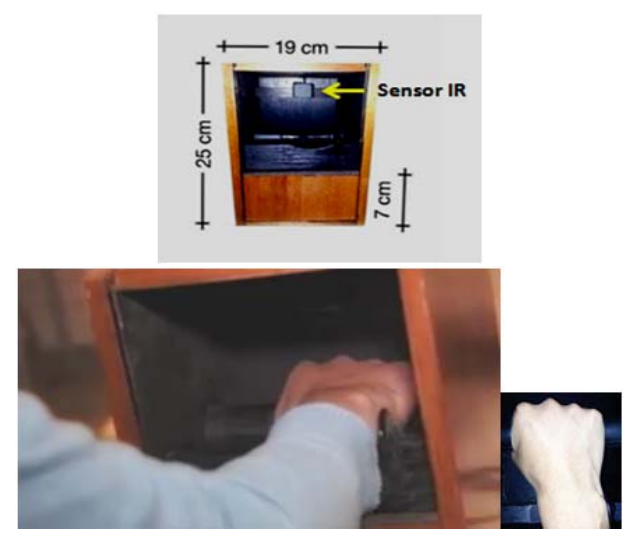

Figura 2. Vistas del recinto de adquisición de imágenes. 
El sensor utiliza un método de captura de imágenes por Infrarrojo-cercano (NIR). El tamaño de la región de adquisición se encuentra entre 40 a $60 \mathrm{~mm}$ por lado. El consumo máximo de potencia es $2.5 \mathrm{~W}$. Las condiciones ambientales para su funcionamiento son: Temperatura (0$\left.60^{\circ} \mathrm{C}\right)$ y Humedad $(10-90 \% \mathrm{RH})$. El tamaño de la imagen capturada por el sensor es de 640 por 480 pixeles en escala de grises, con profundidad de 8bits. La Fig. 3 muestra una imagen adquirida sin aplicar ningún tipo de procesamiento.

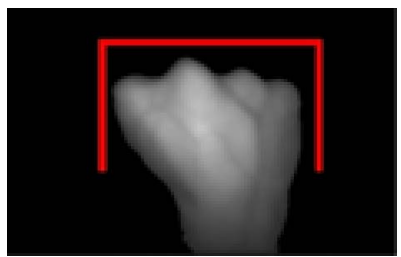

Figura 3. Imagen cruda.

En las siguientes secciones se va a describir consecutivamente las etapas de procesamiento a las cuales es sometida la imagen adquirida. El primer paso es la extracción de la región de interés donde se concentra el mapa de venas del dorso de la mano.

\section{B. Binarización por umbral fijo}

El objetivo de umbralizar o binarizar la imagen adquirida es diferenciar los pixeles de piel de los pixeles correspondientes a la superficie del soporte diseñado.

El procedimiento de binarización es expresado como:

$I_{B}(x, y)=\left\{\begin{aligned} 255, & I(x, y)>\mu_{o} \\ 0, & \text { otra forma }\end{aligned}\right.$

donde $\mathrm{I}(\mathrm{x}, \mathrm{y})$ es la imagen original adquirida (" $\mathrm{x}$ " $\mathrm{e}$ " $\mathrm{y}$ " son las coordenadas espaciales), " $\mu_{o}$ " el umbral de binarización e $\mathrm{I}_{\mathrm{B}}(\mathrm{x}, \mathrm{y})$ la imagen binarizada.

En este caso se utilizó el valor de umbral $\mu_{o}=100$. Este valor fue elegido en función de las condiciones de iluminación establecidas en el recinto, la configuración del sensor de adquisición y los resultados obtenidos en las diferentes pruebas de segmentación de puño. La imagen original binarizada se muestra en la Fig. 4.

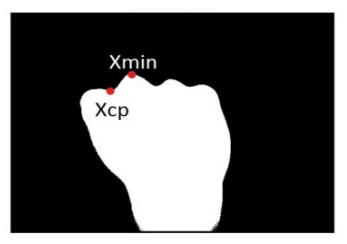

Figura 4. Imagen binarizada.

\section{Eliminación de nudillos}

Después de obtener la imagen binarizada es necesario eliminar los nudillos que conforman el puño de la mano, dado que estos provocan que la región de interés sea muy variable en el momento de su extracción.

El procedimiento para el corte de nudillos es detallado a continuación:

Paso 1: Se ubica el pixel blanco con el menor valor de coordenada " $x$ ". Este pixel puede ser expresado como $\mathrm{I}_{\mathrm{B}}\left(\mathrm{x}_{\min }, \mathrm{y}_{\mathrm{o}}\right)$.

Paso 2: A partir de la fila " $x_{\min }$ " se recorre la imagen hacia abajo (línea por linea), y se mide la longitud horizontal de pixeles blancos continuos. Este procedimiento es realizado hasta que esta longitud supere el umbral $\mu_{1}=200$ (con este valor se obtuvieron los mejores resultados de segmentación ya que se acoge muy bien a la resolución espacial de la adquisición y al tamaño de puño promedio de las personas). La coordenada " $x$ " de esta fila será expresada como " $\mathrm{x}_{\mathrm{cp}}$ ".

Paso 3: Finalmente todas las filas de $I_{B}(x, y)$ desde " $x_{\text {min }}$ " hasta " $\mathrm{x}_{\mathrm{Cp}}$ " son eliminadas, obteniéndose la imagen $\mathrm{I}_{\mathrm{BC}}(\mathrm{x}, \mathrm{y})$ mostrada en la Fig 5.

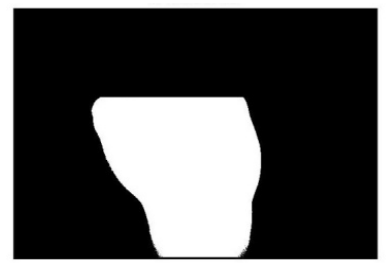

Figura 5. Corte de nudillos.

\section{Segmentación - Extracción de ROI}

La segmentación y extracción de la región de interés (ROI) se desarrolló a través del siguiente procedimiento:

Paso 1: Se establece el tamaño de la región de interés en $M_{R} \times N_{R}$ pixeles. En este caso se ha considerado $M_{R}=150$ y $\mathrm{N}_{\mathrm{R}}=150$ (este tamaño se acoge muy bien a la resolución espacial de adquisición configurada en el sensor).

Paso 2: Se ubica en $I_{B C}(x, y)$ una fila de pixeles en

$\mathrm{x}_{1}=\mathrm{x}_{\mathrm{cp}}+\mathrm{d}_{1}$. Para este caso se consideró $\mathrm{d}_{1}=10$ (este valor de compensación permitió ubicar y centralizar mejor a la región de interés y mejorar el desempeño del algoritmo en la extracción de detalles y en el reconocimiento de usuarios).

Paso 3: A partir de la fila " $x_{1}$ " se recorre la imagen hacia abajo buscando la máxima longitud horizontal de pixeles blancos. Esta longitud será expresada como " $\mathrm{L}_{1}$ ".

Las coordenadas espaciales del primer y último pixel de la fila con longitud " $\mathrm{L}_{1}$ " serán expresados como $\left(\mathrm{x}_{\mathrm{A}}, \mathrm{y}_{\mathrm{A}}\right) \mathrm{y}\left(\mathrm{x}_{\mathrm{B}}, \mathrm{y}_{\mathrm{B}}\right)$ respectivamente.

Paso 4: Se obtiene el vértice superior izquierdo $\left(x_{R 1}, y_{R 1}\right)$ de la ROI como:

$\mathrm{x}_{\mathrm{R} 1}=\mathrm{x}_{\mathrm{A}}$

$\mathrm{y}_{\mathrm{R} 1}=\left(\operatorname{round}\left(\frac{\mathrm{L}_{1}}{2}\right)+\mathrm{y}_{\mathrm{A}}\right)-\operatorname{round}\left(\frac{\mathrm{N}_{\mathrm{R}}}{2}\right)$

donde la función "round(k)" retorna el entero más próximo a "k".

Paso 5: Las otras coordenadas de la ROI serán obtenidas a partir de las siguientes expresiones: 


$$
\begin{aligned}
& \mathrm{x}_{\mathrm{R} 2}=\mathrm{x}_{\mathrm{R} 1} \\
& \mathrm{y}_{\mathrm{R} 2}=\mathrm{y}_{\mathrm{R} 1}+\mathrm{N}_{\mathrm{R}}-1 \\
& \mathrm{x}_{\mathrm{R} 3}=\mathrm{x}_{\mathrm{R} 1}+\mathrm{M}_{\mathrm{R}}-1 \\
& \mathrm{y}_{\mathrm{R} 3}=\mathrm{y}_{\mathrm{R} 1} \\
& \mathrm{x}_{\mathrm{R} 4}=\mathrm{x}_{\mathrm{R} 1}+\mathrm{M}_{\mathrm{R}}-1 \\
& \mathrm{y}_{\mathrm{R} 4}=\mathrm{y}_{\mathrm{R} 1}+\mathrm{N}_{\mathrm{R}}-1
\end{aligned}
$$

Paso 6: Finalmente la ROI es extraída sobre $I(x, y)$ obteniéndose $\mathrm{I}_{\mathrm{R}}(\mathrm{x}, \mathrm{y})$. Esto puede ser expresado como:

$$
\begin{aligned}
\mathrm{I}_{\mathrm{R}}(\mathrm{x}, \mathrm{y})= & \mathrm{I}\left(\mathrm{x}+\mathrm{x}_{\mathrm{R} 1}, \mathrm{y}+\mathrm{y}_{\mathrm{R} 1}\right) \\
& \text { para } 0 \leq \mathrm{x} \leq \mathrm{M}_{\mathrm{R}}-1,0 \leq \mathrm{y} \leq \mathrm{N}_{\mathrm{R}}-1
\end{aligned}
$$

La ROI extraída de I(x,y) se muestra en la Figura 6.
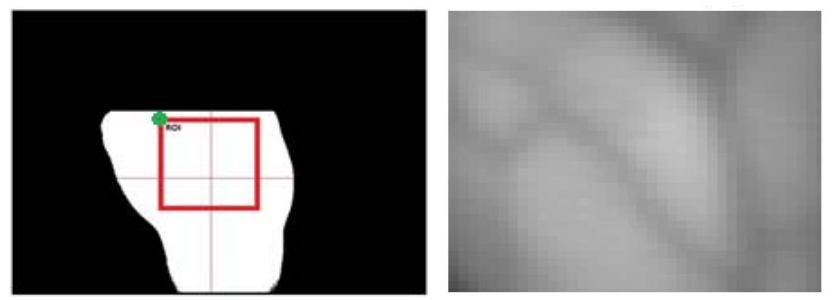

Figura 6. ROI extraída.

\section{E. Binarización de la imagen segmentada}

Para desarrollar el método de extracción del mapa de venas de la región de interés se utilizó el principio propuesto en el método de vecindad de píxeles a distancia [4]. Con ello se logró alcanzar un desempeño bastante satisfactorio en la extracción del mapa requerido y disminuir significativamente la carga computacional del algoritmo.

En la implementación se trabajó con ocho píxeles vecinos a una distancia de 10 píxeles (ancho aproximado de una vena para la resolución de la adquisición) y se realizaron cuatro análisis que generaron 4 imágenes binarias a partir de $I_{R}(x, y)$.

El primer análisis buscó venas en la posición horizontal $\left(\theta=0^{\circ}\right)$. El algoritmo calcula la diferencia entre un determinado pixel ubicado en la posición $(\mathrm{x}, \mathrm{y})$ y los píxeles superiores e inferiores localizados en la dirección vertical a una distancia $\mathbf{d = 1 0}$ pixeles. Si la diferencia obtenida en ambos casos es negativa (ya que el píxel que representa a la vena debe de ser más oscuro en comparación a la piel que la rodea), entonces en esa misma posición espacial (en la imagen binaria resultante) se coloca un valor " 1 ", de lo contrario se coloca un valor " 0 ". De esa manera se genera una imagen binaria que puede ser expresada como:

$I_{R B\left(\theta=0^{\circ}\right)}(x, y)=\left\{\begin{array}{cl}1, & I_{R}(x, y)-I_{R}(x+d, y)<0 \\ & \wedge I_{R}(x, y)-I_{R}(x-d, y)<0 \\ & I_{R}(x, y)-I_{R}(x+d, y) \geq 0 \\ & \wedge I_{R}(x, y)-I_{R}(x-d, y) \geq 0\end{array}\right.$

El segundo $\left(\theta=45^{\circ}\right)$, tercer $\left(\theta=90^{\circ}\right)$ y cuarto análisis $\left(\theta=135^{\circ}\right)$ presentan un planteamiento análogo al primer caso con variaciones en la adición o sustracción de la distancia referida en sus componentes; en ellos se buscaron las venas en otras posiciones acorde a los patrones de identificación ya planteados.

Las imágenes binarias obtenidas para las otras direcciones pueden ser expresadas como:

Para $\theta=45^{\circ}$ :

$I_{R B\left(\theta=45^{\circ}\right)}(x, y)=\left\{\begin{aligned} 1, & I_{R}(x, y)-I_{R}(x+d, y+d)<0 \\ & \wedge I_{R}(x, y)-I_{R}(x-d, y-d)<0 \\ & I_{R}(x, y)-I_{R}(x+d, y+d) \geq 0 \\ 0 & I_{R}(x, y)-I_{R}(x-y, y-d) \geq 0 \\ & \end{aligned}\right.$

Para $\theta=90^{\circ}$ :

$I_{R B\left(\theta=90^{\circ}\right)}(x, y)=\left\{\begin{aligned} 1, & I_{R}(x, y)-I_{R}(x, y+d)<0 \\ & \wedge I_{R}(x, y)-I_{R}(x, y-d)<0 \\ 0, & I_{R}(x, y)-I_{R}(x, y+d) \geq 0 \\ & \wedge I_{R}(x, y)-I_{R}(x, y-d) \geq 0\end{aligned}\right.$

Para $\theta=135^{\circ}$ :

$I_{R B\left(\theta=135^{\circ}\right)}(x, y)=\left\{\begin{aligned} 1, & I_{R}(x, y)-I_{R}(x-d, y+d)<0 \\ & \wedge I_{R}(x, y)-I_{R}(x+d, y-d)<0 \\ 0, & I_{R}(x, y)-I_{R}(x-d, y+d) \geq 0 \\ & \wedge I_{R}(x, y)-I_{R}(x+d, y-d) \geq 0 \\ & \end{aligned}\right.$

Finalmente, la matriz binaria resultante de venas extraídas puede ser expresada como:

$\mathrm{I}_{\mathrm{RB}}(\mathrm{x}, \mathrm{y})=\left\{\begin{array}{cr}255, \quad \forall \mathrm{I}_{0}(\mathrm{x}, \mathrm{y})>0 \\ 0, \forall \mathrm{I}_{0}(\mathrm{x}, \mathrm{y})=0\end{array}\right.$

Donde:

$$
\begin{aligned}
\mathrm{I}_{0}(\mathrm{x}, \mathrm{y})=\mathrm{I}_{\mathrm{RB}\left(\theta=0^{\circ}\right)}(\mathrm{x}, \mathrm{y})+\mathrm{I}_{\mathrm{RB}\left(\theta=45^{\circ}\right)}(\mathrm{x}, \mathrm{y}) \\
+\mathrm{I}_{\mathrm{RB}\left(\theta=90^{\circ}\right)}(\mathrm{x}, \mathrm{y})+\mathrm{I}_{\mathrm{RB}\left(\theta=135^{\circ}\right)}(\mathrm{x}, \mathrm{y})
\end{aligned}
$$

Es importante indicar que antes de la binarización la ROI fue extendida en 10 píxeles adicionales en los bordes, a fin de poder aplicar las condiciones (11), (12), (13) y (14). En la Fig. 7 se muestra un ejemplo de los patrones que se han resaltado a través de la binariazación.

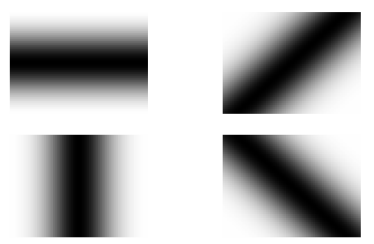

Figura 7. Patrones - binarización.

En la Fig. 8 se muestran los resultados obtenidos en cada una de las etapas que conllevan a la binarización de la ROI. 


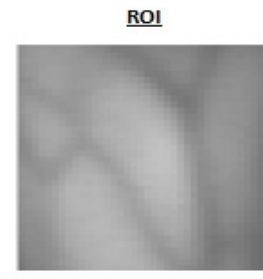

$\underline{\Theta=90}$
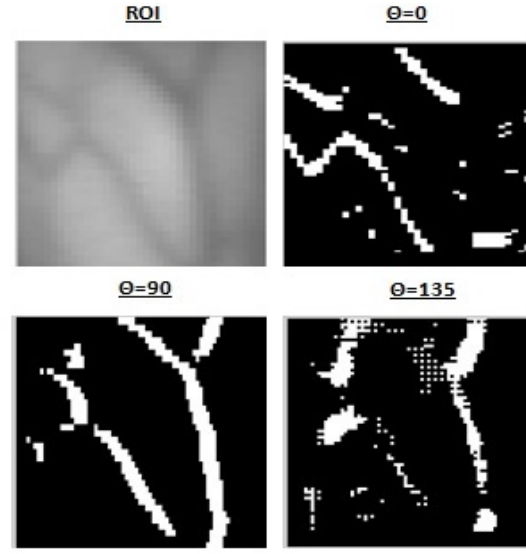

$\underline{\Theta=135}$
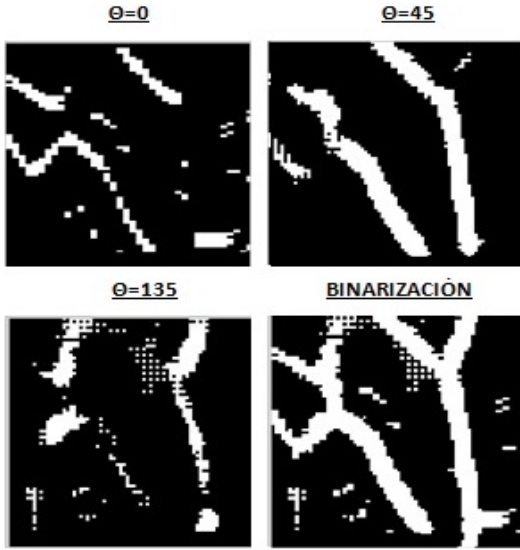

BINARIZACIÓN

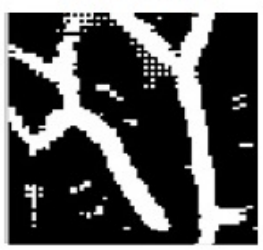

Figura 8. Imágenes del proceso de binarización de la ROI.

\section{F. Extracción de venas principales}

Luego de obtener la binarización de la imagen se procede a filtrar las venas importantes o de mayor presencia. Ello se logra mediante el etiquetado de objetos y el cálculo de áreas de cada etiqueta. El etiquetado de objetos [5] es una función matemática que tiene como objetivo (luego de la binarización de la imagen) la asignación de una etiqueta a todos los píxeles con valor "1" (o 255 para visualización) que se encuentran conectados con vecindad igual 8 .

Después del etiquetado se realiza el conteo de píxeles que conforman una misma etiqueta, a fin de obtener el área de cada objeto. Luego, bajo una condición de umbral, se procede a eliminar las venas secundarias (etiquetas $u$ objetos conformados por un número de pixeles menor a un umbral establecido). El valor del umbral utilizado en este caso fue de 20 pixeles. Con este valor se logró eliminar gran parte de objetos pequeños (considerados como ruido o artefactos) que comprometían el desempeño del algoritmo propuesto. Fueron realizadas varias pruebas para poder determinar el valor de umbral indicado.

Después de obtenida la imagen denominada venas principales (véase en la Figura 9), es necesario realizar el proceso de dilatación. Este proceso se realiza con el objetivo de evitar la posible presencia de ramificaciones no deseadas en la esqueletización; con ello se logrará una mejor visualización de la posición de las venas y corroborar el correcto funcionamiento del algoritmo de binarización.

Para el proceso de dilatación se realiza un barrido de la imagen basado en el rellenado de píxeles en la vecindad 8. Los píxeles serán asignados como negros o blancos dependiendo del predominio de color en la vecindad. Ello puede causar pequeños puntos negros en el interior de las venas, sin embargo estos son corregidos mediante la etiqueta de objetos y la detección de las áreas que representan respecto al fondo negro de la imagen.

\section{G. Esqueletización}

Posterior a la obtención de la etapa final del proceso de binarización de la región de interés (véase la Figura 9) se realiza la esqueletización de la imagen a través del algoritmo de Pavlidis [6] (véase la Fig. 10). Se trata de un algoritmo morfológico que describe la forma de una imagen en la menor cantidad de píxeles.

Los píxeles sujetos a esqueletización son sólo aquellos que presentan similitud con algunas plantillas del algoritmo [6].

La Fig. 11 muestra una superposición entre la imagen resultante de la esqueletización y la imagen original de la ROI extraída.

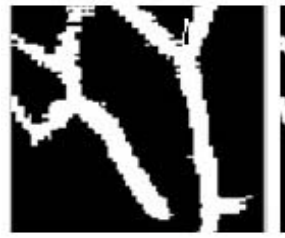

(a)

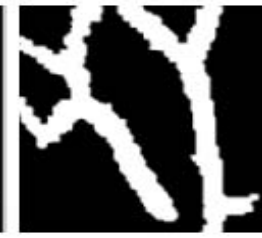

(b)
Figura 9. (a) Venas principales y (b) Binarización final con dilatación.

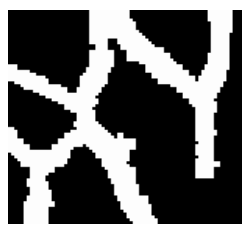

(a)

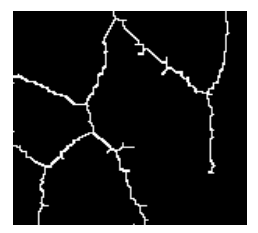

(b)
Figura 10. (a) Venas Principales y (b) Esqueletización.

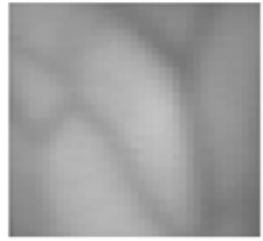

(a)

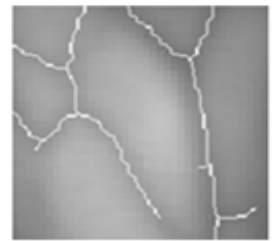

(b)
Figura 11. (a) ROI original extraída y (b) ROI superpuesta con la esqueletización.

\section{H. Generación de la base de datos}

La base de datos es generada a partir del registo de tres imágenes capturadas por cada usuario. Cada imagen se procesa y se almacena en el formato de venas principales binarizadas y dilatadas Al actualizar la base de datos, el sistema procesará todas las imágenes almacenadas y extraerá las ROI de cada una de ellas. Luego las procesa hasta la etápa de extracción de venas principales. 


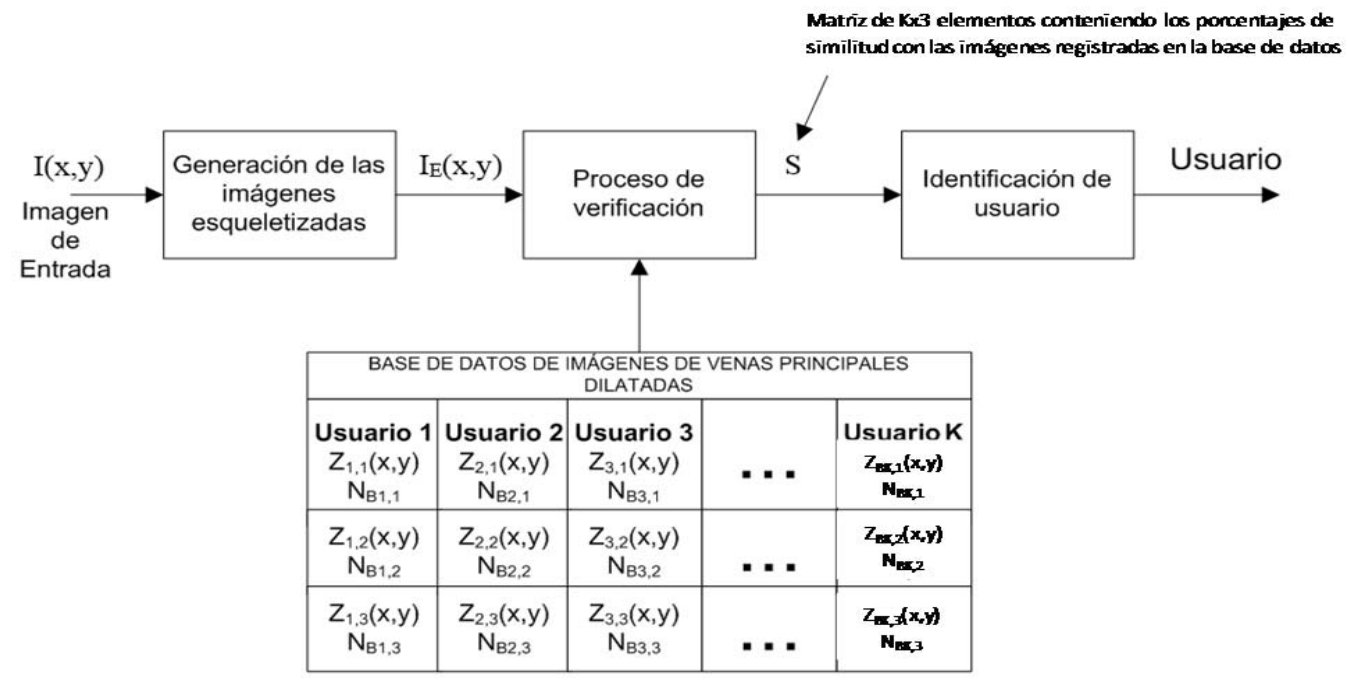

Figura 12. Proceso de verificación de usuarios.

\section{Verificación del usuario}

El proceso de verficación implica determinar el nivel de semejanza entre una imagen adquirida esqueletizada y las imágenes de la base de datos (ver Fig. 12).

Esto es realizado entre la imagen esqueletizada $\mathrm{I}_{\mathrm{E}}(\mathrm{x}, \mathrm{y})$ obtenida la imagen adquirida $\mathrm{I}(\mathrm{x}, \mathrm{y})$ y las imágenes dilatadas de cada usuario $\mathrm{Z}_{\mathrm{i}, \mathrm{j}}(\mathrm{x}, \mathrm{y})$ almacenadas en la base de datos (ver Fig. 13).

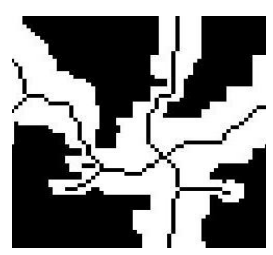

Figura 13. Imagen esqueletizada superpuesta a la imagen dilatada.

El proceso implica obtener en primer lugar la matriz de porcentaje de correlación cruzada (para diversas traslaciones de $I_{E}(x, y)$ ) definida como:

$\mathrm{R}_{\mathrm{i}, \mathrm{j}}(\mathrm{m}, \mathrm{n})=\frac{100}{\mathrm{~N}_{\mathrm{B}_{\mathrm{i}, j}}} \cdot \sum_{\mathrm{x}=0}^{149} \cdot \sum_{\mathrm{y}=0}^{149} \mathrm{z}_{\mathrm{i}, \mathrm{j}}(\mathrm{x}, \mathrm{y}) \mathrm{I}_{\mathrm{E}}(\mathrm{x}+\mathrm{m}, \mathrm{y}+\mathrm{n})$

para $i=1,2, . ., K ; j=1,2,3 ; m=-5,0,5 ; n=-5,0,5$

donde $\mathrm{I}_{\mathrm{E}}(\mathrm{x}, \mathrm{y})$ es la imagen adquirida, procesada $\mathrm{y}$ esqueletizada en sus venas principales; $\mathrm{Z}_{\mathrm{i}, \mathrm{j}}(\mathrm{x}, \mathrm{y})$ es la $\mathrm{j}$-ésima imagen almacenada y dilatada en venas principales del usuario “i”. Finalmente $\mathrm{N}_{\mathrm{B}_{\mathrm{i}, \mathrm{j}}}$ es el número de pixeles blancos de la $\mathrm{j}$ ésima imagen almacenada y dilatada en venas principales correspondiente al usuario " $\mathrm{i}$ ".

Las traslaciones de diversas imágenes esqueletizadas $I_{E}(x, y)$ sobre una imagen de la base de datos $Z_{i, j}(x, y)$ para el cálculo de la correlación cruzada son mostradas en la Fig. 14.
El resultado del proceso de verificación es la matriz $\mathbf{S}$ definida como:

$\mathbf{S}=\left[\begin{array}{ccc}\beta_{1,1} & \beta_{1,2} & \beta_{1,3} \\ \beta_{2,1} & \beta_{2,2} & \beta_{2,3} \\ : & : & : \\ \beta_{\mathrm{K}, 1} & \beta_{\mathrm{K}, 2} & \beta_{\mathrm{K}, 3}\end{array}\right]$

Donde los valores $\beta_{\mathrm{i}, \mathrm{j}}$ satisfacen la siguiente condición:

$$
\begin{aligned}
& \beta_{i, j} \geq R_{i, j}(m, n), \\
& \text { para } m=-5,0,5 \wedge n=-5,0,5
\end{aligned}
$$
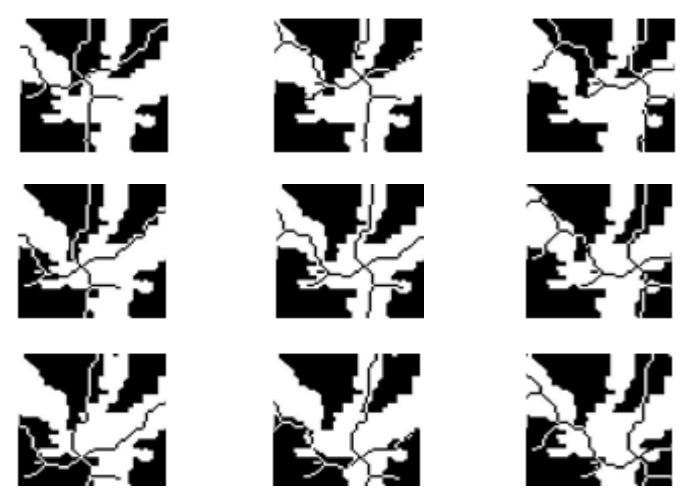

Figura 14. Traslaciones de imágenes esqueletizadas de entrada $I_{E}(x, y)$ sobre una imagen $\mathrm{Z}_{\mathrm{i}, \mathrm{j}}(\mathrm{x}, \mathrm{y})$ de la base de datos correspondiente a un determinado usuario.

\section{J. Identificación del usuario}

A partir de $\mathbf{S}$ se identifica el valor $\beta_{\mathbf{i}_{0}, j_{0}}$ que satisface la siguiente condición:

$\beta_{\mathrm{i}_{0}, \mathrm{j}_{0}} \geq \beta_{\mathrm{i}, \mathrm{j}}$ para $\mathrm{i}=1,2, . ., \mathrm{K} \wedge \mathrm{j}=1,2,3$ 
Si $\beta_{\mathrm{i}_{0}, \mathrm{j}_{0}}$ es mayor a $90 \%$, entonces se valida y se identifica al usuario ubicado en la posición " $\mathrm{i}_{\mathrm{o}}$ " de la base de datos; de lo contrario se solicita volver a intentar con una nueva captura.

\section{RESUltados}

La Tabla II muestra los resultados que se obtuvieron de pruebas totalmente aleatorias con una población heterogénea de 50 personas (32 hombres y 18 mujeres entre las edades de 14 y 78 años). Por cada persona registrada se realizaron 30 verificaciones. Cabe resaltar que cada usuario tuvo una charla de orientación previa de 5 minutos para el correcto uso del sistema.

\begin{tabular}{|c|c|c|c|c|c|c|}
\hline \multirow[b]{2}{*}{ Usuarios } & \multirow[b]{2}{*}{$\begin{array}{c}\text { Intentos } \\
\text { por } \\
\text { usuario }\end{array}$} & \multirow[b]{2}{*}{$\begin{array}{c}\text { Total } \\
\text { de } \\
\text { pruebas }\end{array}$} & \multicolumn{4}{|c|}{ Resultados } \\
\hline & & & $\begin{array}{c}\text { Falso } \\
\text { rechazo } \\
\text { (FRR) }\end{array}$ & $\begin{array}{c}\text { Falsa } \\
\text { aceptación } \\
\text { (FAR) }\end{array}$ & $\begin{array}{c}\text { FRR } \\
(\%)\end{array}$ & $\begin{array}{c}\text { FAR } \\
(\%)\end{array}$ \\
\hline 50 & 30 & 1500 & 7 & 0 & 0.47 & 0.00 \\
\hline
\end{tabular}

El tiempo promedio de respuesta del sistema fue de 1.8 segundos (luego de la captura de la imagen). Para este caso se utilizó una laptop con procesador Intel Core i5 - 2520M $2.50 \mathrm{GHz}$ y memoria RAM de 4GB. Dicho tiempo fue obtenido calculando la media aritmética de 50 procesos aleatorios de verificación con 50 usuarios registrados. Cabe mencionar que el tiempo de respuesta es variante según la complejidad de las venas del dorso de la mano (debido a la cantidad de recorridos cíclicos a realizar por el programa en la etapa de esqueletización) y la cantidad de personas registradas en la base de datos.

Los resultados obtenidos posteriores a la validación del sistema fueron comparados con otras tecnologías similares en objetivo (ver Tabla III).

TABLA III. COMPARATIVO CON OTRAS TECNOLOGÍAS BIOMÉTRICAS

\begin{tabular}{|c|c|c|c|c|}
\hline Tecnología & $\begin{array}{c}\text { Medio de } \\
\text { Análisis }\end{array}$ & $\begin{array}{c}\text { FAR (\%) } \\
\text { (promedio) }\end{array}$ & $\begin{array}{c}\text { FRR (\%) } \\
\text { (promedio) }\end{array}$ & $\begin{array}{c}\text { Tiempo } \\
\text { Promedio de } \\
\text { Procesamiento } \\
\text { (Segundos) }\end{array}$ \\
\hline $\begin{array}{c}\text { Fujitsu - } \\
\text { Palm Vein } \\
\text { Reader }\end{array}$ & $\begin{array}{c}\text { Venas de } \\
\text { la Palma } \\
\text { de la } \\
\text { mano }\end{array}$ & 0.0008 & 0.01 & 0.8 \\
\hline $\begin{array}{c}\text { Imagen del } \\
\text { Iris }\end{array}$ & $\begin{array}{c}\text { Iris } \\
\text { ocular }\end{array}$ & 0.03 & 0.9 & $\approx 4$ \\
\hline $\begin{array}{c}\text { Impresión } \\
\text { de Huella } \\
\text { Dactilar }\end{array}$ & $\begin{array}{c}\text { Huella } \\
\text { Dactilar }\end{array}$ & 0.059 & 1.33 & $\approx 0.2$ \\
\hline $\begin{array}{c}\text { Sistema } \\
\text { Propuesto }\end{array}$ & $\begin{array}{c}\text { Venas } \\
\text { dorso de } \\
\text { la mano }\end{array}$ & $\mathbf{0 . 0 0 0 0}$ & $\mathbf{0 . 4 7}$ & $\mathbf{1 . 8}$ \\
\hline
\end{tabular}

El sistema propuesto tiene una FAR de cero, siendo necesario incrementar la base de datos para obtener resultados más certeros, no obstante la FRR es mayor comparado al sistemas desarrollado por Fujitsu [6].

La Tabla III [7], [8], [9] evidencia un desempeño cuantitativo muy satisfactorio del método propuesto respecto a las otras tecnologías biométricas (analizados en varios aspectos).

\section{CONClusiones y Mejoras Futuras}

Se logró desarrollar un sistema biométrico constituido por un módulo de adquisición de imágenes infrarrojas y un aplicativo de software de reconocimiento de patrones capaz de realizar las tareas de captura, registro y validación de personas utilizando los patrones de la red vascular del dorso de la mano. Los métodos desarrollados para los procesos de extracción de ROI, binarización y verificación han presentado un eficiente desempeño para tal finalidad.

La extracción de la ROI permitió estabilizar errores de desplazamiento tanto vertical, como horizontal encontrándose aún problemas en posibles giros angulares del puño. Sin embargo, las correcciones realizadas, permitieron aplicar métodos de procesamiento de imágenes y, con ello, obtener la visibilidad de las venas ubicadas en el dorso de la mano.

El método de binarización planteado es eficiente, ya que a pesar de la heterogeneidad de la imagen se logra obtener un mapa binarizado donde se obtiene la posición de las venas. El utilizar otros métodos de binarización ya sea por umbral o el método de OTSU generan problemas debido a que las venas no poseen un mismo nivel de grises en todo el espacio de adquisición. El método de OTSU adaptivo logra resaltar significativamente la posición de las venas; sin embargo genera marcos indeseados al ser adaptado por bloques debido a la heterogeneidad de la imagen.

El proceso de dilatación es efectivo para la aplicación requerida ya que permite obtener un correcto trazo de las venas aplicando pequeñas correcciones.

La experiencia del usuario en el proceso de adquisición de la imagen es de suma importancia para registrar nuevos patrones correctamente y permitir que la validación e identificación sean efectivas y eficientes.

Como mejora a futuro debe considerarse el incremento de la eficiencia de los algoritmos de procesamiento. Esto con el objetivo de aminorar la carga computacional y el tiempo de procesamiento. Asimismo es importante realizar un método de descarte ante la validación de patrones, a fin no presentar problemas de elevados tiempos de procesamiento para más de 50 usuarios registrados.

Finalmente debe considerarse dar movimiento vertical al sensor de adquisición. Ello se debe a que se presentan diversas medidas palmar-dorsal en cada usuario y es necesaria una distancia óptima para realizar la captura de la imagen; dicha mejora logrará contribuir con la ergonomía del usuario.

\section{AgRADECIMIENTOS}

Los autores agradecen a todas aquellas personas que se ofrecieron voluntariamente para ayudar a validar el sistema propuesto y obtener los resultados presentados en el presente documento.

\section{REFERENCIAS}

[1]R. SAINI and N. RANA, "Comparison of Various Biometric Methods", International Journal of Advances in Science and Technology (IJAST), Vol. 2, March 2014. 
[2]O. Motato y H. Loaiza, "Identificación biométrica utilizand o imágenes infrarrojas de la red vascular de la cara dorsal de la mano" - en la Revista Ingeniería e Investigación Vol 29. No. 1, Abril de 2009

[3] H. Chen, G. Lu and R. Wang, "A New Palm Vein Matching Method Based on ICP Algorithm", 2009.

[4]Y. Zhang, Q. Li, J. You and P. Bhattacharya, "Palm Vein Extraction and Matching for personal Autentification" - Universidad KLN, Hong Kong, 2001.

[5]

http://www.tsc.uc3m.es/imagine/Curso_ProcesadoMorfologico/Contenido/ Operaciones/OperacionesMorfologicas.html

[6]T. Pavlidis, "A thinning Algorithms for Discrete Binary Images, Computer Graphics and Image Processing", pp. 142-157, 1980.

[7] FUJITSU Computer Products, Inc., Palm Vein Authentication. 2006.

[8]P. S. Revankar and A. Anjum, "Performance Analysis of Iris Authentication System in Wavelet Domain", International Journal of Advanced Research in Electrical, Electronics and Instrumentation Engineering, Vol. 2, pp. 4821-4828, October 2013.

9]FEDERAL OFFICE FOR INFORMATION SECURITY (Germany), Study: "Evaluation of Fingerprint Recognition Technologies - BioFinger", Final Report, August 2004.

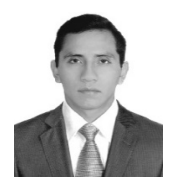

Willy Alejo, Ingeniero Electrónico de la Universidad Peruana de Ciencias Aplicadas (UPC) con estudios de postgrado a nivel de diplomado en Derecho de la Construcción, Contrataciones Estatales, Gestión de Proyectos y Comercio Exterior. Ex - Director de Proyectos y Co - Fundador del Centro de Desarrollo de Aplicaciones Tecnológicas APPTECH - Lima. Actualmente candidado al Master Bussines Administration (MBA) del Programa de Alta Dirección (PAD - UDEP). Su área de investigación está enfocada en el procesamiento de señales e imágenes digitales

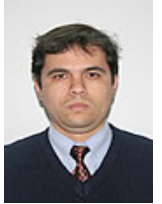

Guillermo Kemper, recibió en 1994 el título de Ingeniero Electrónico de la Universidad Privada Antenor Orrego de Trujillo, Perú. En 1996 obtuvo el grado de Maestro de la Universidad Estadual de Campinas - UNICAMP, Brasil. En el 2001 obtuvo el titulo de doctor en Ingeniería Eléctrica y Comunicaciones también de la UNICAMP. Ha participado durante mas de 3 años del Convenio CPqD (Centro de Pesquisa y Desenvolvimiento de Telebrás) - UNICAMP, desarrollando trabajos orientados al diseño, simulación y evaluación de codificadores de audio y vídeo. Actualmente es profesor investigador de los programas de pre-grado y post-grado de las Escuelas Ingeniería Electrónica de la Universidad de San Martín de Porres de Lima - Perú (USMP) y de la Universidad Peruana de Ciencias Aplicadas (UPC). Asimismo es investigador del Instituto Nacional de Investigación y Capacitación en Telecomunicaciones del Perú - INICTEL-UNI. Sus áreas de investigación son codificación de voz, audio, imágenes y vídeo; así como comunicaciones digitales y televisión digital.

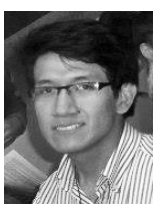

Daniel Rodríguez, Ingeniero Electrónico de la Universidad Peruana de Ciencias Aplicadas (UPC). Tiene interés por la investigación en áreas como: procesamiento de imágenes y señales, bioingeniería, automatización y teoría de control. 